\title{
The Design and Implementation of CNC System for Near-field Electrospinning
}

\author{
Chen MEI ${ }^{1}$, Han WANG ${ }^{1 *}$, Xin-Du CHEN ${ }^{1}$, Yuan-Xiang XIA ${ }^{1}$, Zun-Wei WEN ${ }^{1}$, \\ Ze-Feng HUANG ${ }^{1}$, Yao-Bin ZENG ${ }^{2}$, Zhi-Jin LI' ${ }^{2}$, Guo-Rui HE ${ }^{2}$ \\ ${ }^{1}$ Department of Electromechanical Engineering, Guangdong University of Technology, \\ Guangzhou, 510006, China \\ ${ }^{2}$ Foshan Supervision Testing Centre of Quality and Metrology, China \\ wanghangdut@126.com \\ ${ }^{*}$ corresponding author
}

\begin{abstract}
Keywords: Near-Field Electrospinning NFECNC system; micro/nano structure; automatic graphic programming
\end{abstract}

\begin{abstract}
Near-Field Electrospinning(NFES) is a novel method to fabricate aligned micro/nano structure. At present, the bottleneck of restraints on realizing industrial application for NFES is that direct writing device can't meet its requirements in both stability and precision.This technology is mainly used in biomedicine, flexible electronic circuits and MEMS devices and manufacture of grating mold ruler, etc. In order to realize fabricating the patterned micro/nano structure stably and getting high-precision deposition position by NFES, the four-axis CNC system is set up, and its hardware and software design, pre-designed track of motion platform, the deposition position precision are investigated.The CNC system software is developed based on QT4.8 development tool and realizes the automatic graphic programming of patterned micro/nano structure. Experimental results show that the four-axis $\mathrm{CNC}$ system is suitable to fabricate the patterned micro/nano structure, and the precision of the deposition position of nanofiber is $5 \mu \mathrm{m}$. This CNC system can satisfy the requirements of fabricating aligned micro/nano structure by NFES. The CNC system establishes the fundamention for the industrial application of NFES.
\end{abstract}

\section{Introduction}

The Nanofiber is a new functional material, which has important value and broad applied prospect in many fields, such as microsensor, electronic devices, organic flexible electronic, biological tissue[1],etc. As an effective method to produce nanofibers, the electrospinning owns unique superiority that meets the requirement of development such as low cost, fast and convenient for manufacturing 2D micro-nano structure, so there are more and more researchers studying the electrospinning. Electrospinning originateing from electrohydrodynamic theory is a simple and significant way to fabricate nanofiber, However, the widespread method of electrospinning leads into random deposition of electrospun nanofiber because of the bending instability of jet, which restricts the development and application of the electrospinning technology. Near-Field Electrospining(NFES) overcomes the bending instability and uncontrol of jet in conventional electrospinning by shorting the distance between spinneret and collector[2,3], and at the same time, the obvious superiority of narrow linewidth, high speed and high precision that makes the NFES to fabricate orderly nanofiber, which extends the application range of NFES[4,5].

The patterned micro/nano structure can be fabricated by controlling the motion trajectory and the velocity of collector[6]. The one of key point of Near-Field Electrospining (NFES) is how to design the four-axis CNC system[7], and the location accuracy and interpolation algorithm of fouraxis CNC system is the bottleneck to realize the industrialization of NFES[8,9].

This paper is armed to disign and implement the four-axis CNC system for Near-Field Electrospining(NFES), which is based on GOOGOL TECHNOLOGY GTS four-Axis control card, linear motors and servo system[10]. The CNC system software, path planning and the depositing accuracy are investigated. 


\section{The framework of CNC system}

The open and modular structure of "IPC+motion control card" is applied in this control system.The structure of the CNC system is shown in Fig.1. As the upper computer, the IPC realizes the compound and management of system, human-computer interaction, real-time process input, compilation and simulation,etc. As the control core, the four-Axis motion control card of GOOGOL TECHENOLOGY is applied in this system to achieve high-precision control of closed-loop, and the precision requirements of fabricating the patterned micro/nano structure will be guaranteed. The scheme combines the advantages of both and ensures the stability, reliability and accuracy of the CNC system.In addition,it possess high openness and provides convenience for its updating and maintaining.

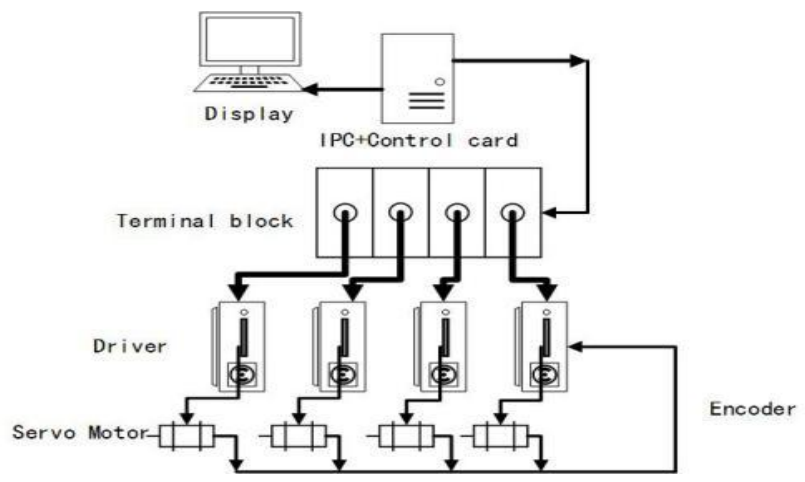

Fig. 1 The structure of the CNC system

\section{Hardware of the CNC system}

The four-Axis motion platform consists of three axis of Cartesian coordinates and one $\theta$-Axis of rotating around Z-Axis. Fig.2. shows the 3D motion module and Drivers. The hardware selection of motion platform plays a crucial role in overall performance of the whole equipment. As the control core of the motion system, the GTS four-axis motion control card is able to achieve closed-loop control with high precision and guarantee the accuracy of 2D micro-nano structure. The X-Axis has a high request for precision,so the linear motor of Kollmorgen DDL motor and driver is selected, and the resolution of this motor is up to $4 \mu \mathrm{m} /$ pulse without subdividing. Y-Axis does not have high request for localization accuracy but requests fast reaction and straightness accuracy, so the linear motor of SURUGA and 5-Phase Stepping Motor Unit is selected. As for Z-Axis and $\theta$-Axis, they have no high request for accuracy and respond speed, so step motor of ORENTAL MOTOR and step motor driver are selected. The RENISHOW grating ruler is selected as X-Axis feedback device, the resolution of which is up to $0.1 \mu \mathrm{m}$. The motors of Y-Axis,Z-Axis and $\theta$-Axis are provided with rotary encoder. The hardware of the CNC system meets the demands for accuracy and reliability of NFES.

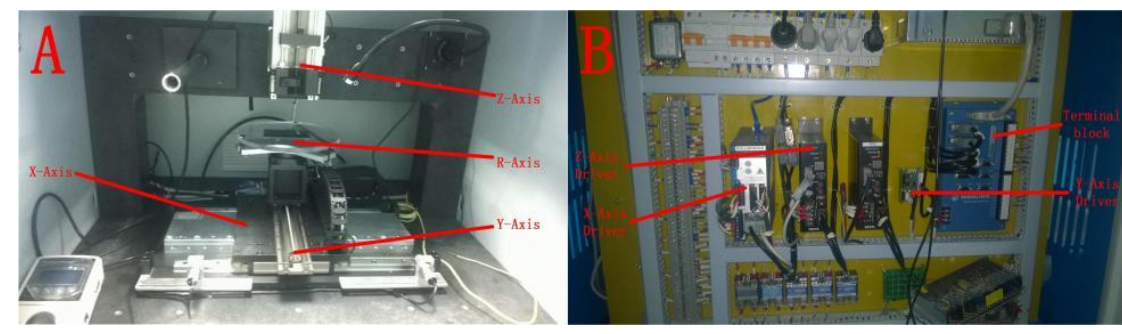

Fig. 2 3D motion module and drivers

\section{Software of the CNC system}

The software of control system is developed on the basis of QT platform within VC++ and dynamic linkbase provided by GTS motion control card under operating system of Windows. Based 
on the modularized and object-oriented architecture, the software control system is designed to meet the demands for the function of 2D micro-nano structure in all direction[5]. This software includes four modules mainly, the division of modules is indicated as Fig. 3.

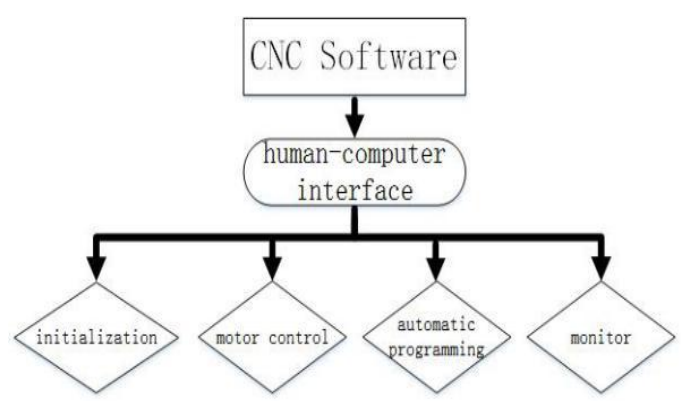

Fig. 3 The division of software modules

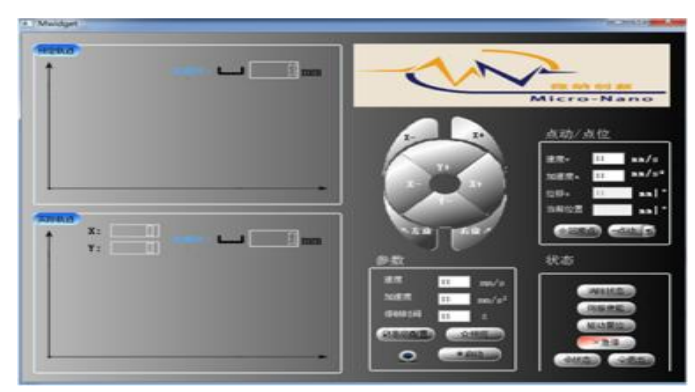

Fig. 4 Human-computer main interface

The initialization module of software is used to set up the initialization parameter of the controller. The motor control module is used to send motor controlling procedure to motion control card, and then motion control card calls corresponding controlling instruction[7]. The automatic programming module is used to extract the points of pattern and translate into command language code. The monitor module is applied to feedback the location information and state information of the track[6]. The human-machine main interface is designed to meet human-machine interaction and the interface is indicated as Fig.4.

\section{Experiments}

The experiments are to test the performance of the CNC system. Fig.5 and Fig.6 show the comparison test between designated location and deposition position of jet. The Fig.5 is the parallel line pattern and the test result shows that the repositioning resolution meets the demands of $2 \mathrm{D}$ micro-nano structure.

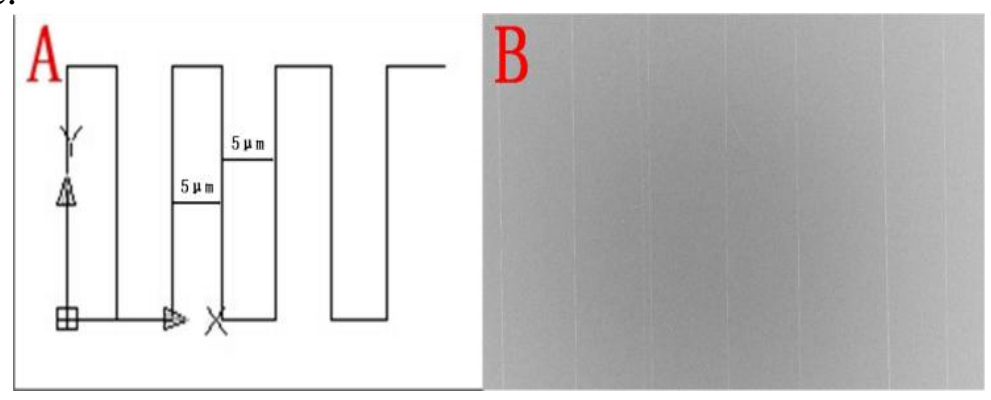

Fig. 5 The direct-written parallel line pattern(motion speed $120 \mathrm{~mm} / \mathrm{s}$ )
A: designated location.
B: deposition position of jet

The Fig. 6 is the arrows pattern and the test result shows that the CNC system realizes the automatic graphic programming of $2 \mathrm{D}$ structure. 


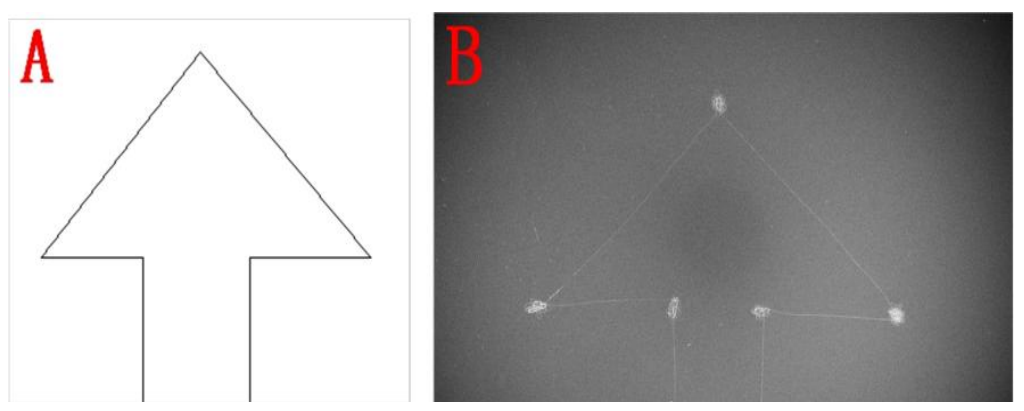

Fig.6.The arrows pattern by automatic programming(motion speed $5 \mathrm{~mm} / \mathrm{s}$ )

A designed CAD drawing.

B deposition position of jet.

\section{Conclusions}

Based on the characteristics of high stability and precision on the NFES process, the four-axis CNC system consists of hardware and software is designed in this paper. The hardware is conposed by motion control card, linear motor and AC sevor motor. The software is developed based on QT platform by $\mathrm{C}++$ and the software includes four modules of initialization, motor control, automatic programming and monitor. The comparison experiments on the CNC system between designated location and deposition position of jet show that the CNC system meets the requirments of highprecision deposition position by NFES and realizes the automatic graphic programming of patterned micro/nano structure. The CNC system is used to fabricate grating mold ruler and provides an excellent way to improve the industrial application of micro/nano structure.

\section{Acknowledgments}

This work was financially supported by Project of Science and Technology of Guangdong Province, China(No.2013B011301005), Special Project on the Integration of Industry, Education and Research of Dong Guan (No.2013509109101), Key Project of Science and Technology of Guangdong Province, China (No.2015B010124001), National Natural Science Foundation of China (No.51305084), Guangdong Natural Science Foundation (Grant Nos.2015A030312008, No.2015B010102014), Training program for outstanding young teachers in higher education institutions of Guangdong Province (No.YQ2015056), Guangdong Science and Technology Plan (Grant Nos.2015B010104006), and Outstanding young scholar in science and technology innovation(No.2014TQ01X212).

\section{References}

[1] Xie Jingwei, Liu Wenying, Matthew $\mathrm{R}$ MacEwan. Tissue engineering: nanofiber membranes with controllable microwells and structural cues and their use in forming cell microarrays and neuronal networks[J].Small, 2011, 7 ( 3): 285-290.

[2] Sun D. H., Chang C., Li S., Lin L. W. Near-field electrospinning [J]. Nano Letters, 2006,6(4): 839-842.

[3] G. F. Zheng, L. Y. Wang, D. H. Sun, Micro/Nano-Structure Direct-Write Technology Based on Near-Field Electrospinning, Nanotechnology and Precision Engineering, 6 (2008) 20-23.

[4] Wu Rong-Hua, Tsai Ming-Jer, Ho Ko-Shan, Wei Ting-En, Hsieh Tar-Hwa, Han Yu-Kai, Kuo Chung-Wen, Tseng Po-Hao, Wang, Yen-Zen. Sulfonated polyaniline nanofiber as Pt-catalyst conducting support for proton exchange membrane fuel cell [J]. Polymer, 55(8): 2035-2043, 2014. 
[5] Kijeńska Ewa, Prabhakaran Molamma P, Swieszkowski Wojciech, Kurzydlowski Krzysztof J, Ramakrishna Seeram. Interaction of Schwann cells with laminin encapsulated PLCL core-shell nanofibers for nerve tissue engineering [J]. European Polymer Journal, 50: 30-38, 2014.

[6] Koga Hirotaka, Nogi Masaya, Komoda Natsuki, Nge Thi Thi, Sugahara Tohru, Suganuma Katsuaki. Uniformly connected conductive networks on cellulose nanofiber paper for transparent paper electronics [J]. NPG Asia Materials, 6(3): e93, 2014.

[7] Jin Hai-Fei,Ding Wen-Zheng,Wang Mu-Lan.Design of Controlling System for NC Worktable Based on Motion Control Card(In Chinese).Development \&Innovation of Machine \&Electrical Products.2012.

[8] Ruizhi Rao,Design and Implementation of four -axis control grinding plate(In Chinese).2011.

[9] Geng HongTao,ShuZhiBing,Inveestigation of the Software Exploitation Technique for a Control System Based on a Multi-axis Motion Control Card in VC.MACHINE TOOL\&HIDRAULICS.2007.

[10] Liu Chang-hong,Song Peng,Liu Yan-Chun,Tang Jian-Bo,Research on Application of a Numerical Control System Based on Motion Control Card.Journal of Dalian Nationalities University.2010. 